\title{
Quasi-steady state power law model for flow of (La0.85Sr0.15)0.9MnO3 ceramic slurry in tape casting
}

\author{
Jabbari, M; Bulatova, R; Hattel, J H; Bahl, C R H
}

Published in:

Materials Science and Technology

Link to article, DOI:

10.1179/1743284713Y.0000000255

Publication date:

2013

Document Version

Peer reviewed version

Link back to DTU Orbit

Citation (APA):

Jabbari, M., Bulatova, R., Hattel, J. H., \& Bahl, C. R. H. (2013). Quasi-steady state power law model for flow of $\left(\mathrm{La}{ }_{\mathrm{Sr}} \mathrm{Sr}\right)_{10} \mathrm{MnO}_{3}$ ceramic slurry in tape casting. Materials Science and Technology, 29(9), 1080-1087. https. 9 Foloi.8irg/10.9179/\$743284713Y.0000000255

\section{General rights}

Copyright and moral rights for the publications made accessible in the public portal are retained by the authors and/or other copyright owners and it is a condition of accessing publications that users recognise and abide by the legal requirements associated with these rights.

- Users may download and print one copy of any publication from the public portal for the purpose of private study or research.

- You may not further distribute the material or use it for any profit-making activity or commercial gain

- You may freely distribute the URL identifying the publication in the public portal 


\title{
Quasi-steady state power law model for flow of $\left(\mathrm{La}_{0.85} \mathrm{Sr}_{0.15}\right)_{0.9} \mathrm{MnO}_{3}$ ceramic slurry in tape casting
}

\author{
M. Jabbari*1 ${ }^{1}$, R. Bulatova ${ }^{2}$, J. H. Hattel ${ }^{1}$ and C. R. H. Bahl ${ }^{2}$
}

One of the most common ways used to produce multilayer ceramics is tape casting. In this process, the wet tape thickness is one of the single most determining parameters affecting the final properties of the product, and it is therefore of great interest to be able to control it. In the present work, the flow in the doctor blade region of a slurry containing $\left(\mathrm{La}_{0} \cdot{ }_{85} \mathrm{Sr}_{0 \cdot 15}\right)_{0.9} \mathrm{MnO}_{3}(\mathrm{LSM})$ material is described with a simple quasi-steady momentum equation in combination with an Ostwald-de Waele power law constitutive equation. Based on rheometer experiments, the constants in the Ostwald-de Waele power law are identified for the considered LSM material and applied in the analytical solution for the tape thickness. This solution is then used for different values of substrate velocity and doctor blade height and compared with experimental findings of the wet tape thickness, and good agreement is found.

Keywords: Tape casting, Doctor blade, Fluid flow, Non-Newtonian, Power law

\section{Introduction}

Tape casting is an important process for producing large area, thin, flat ceramics, which can be patterned, stacked and laminated to form three-dimensional structures. ${ }^{1}$ The method was originally developed for producing electronic ceramics (insulating substrates and packages and multilayer capacitors) and is still mainly used for this. Structural laminates, knives, membranes and solid oxide fuel cells are examples of other applications for thin ceramics formed by tape casting. The tape thickness that can be achieved is generally in the range of 25 up to $1 \mathrm{~mm}$, but it is possible to produce tapes with thicknesses of $<5 \mu \mathrm{m}$.

The parallel (doctor) blade process was first used in preparing ceramic tapes in the 1940s, and it has a key role in producing thin and flat ceramic tapes.,3 Thickness control is of critical importance in tape casting, since it affects the final properties of the tape. Different parameters such as powder distribution, slurry composition, flow field and sintering affect the thickness of the final tape. ${ }^{4-6}$ In addition to this, the geometry of the process set-up itself has different effects on the related final properties of the manufactured product. ${ }^{7}$

In the tape casting process, a slurry is pumped into a reservoir and this slurry is then moved by the peeling belt. Since this technique is used to produce tapes with relatively small thicknesses, accurate control of the tape

${ }^{1}$ Department of Mechanical Engineering, Technical University of Denmark, Nils Koppels Allé, Kgs. Lyngby 2800, Denmark

${ }^{2}$ Department of Energy Conversion and Storage, Technical University of Denmark, Frederiksborgvej 399, Building 779, Roskilde, Denmark

*Corresponding author, email mjab@mek.dtu.dk thickness which exits the doctor blade is a key factor determining the final properties.

One of the most challenging parts of modelling the flow of the ceramic slurry in the tape casting process is to conduct the modelling with the proper constitutive equation which governs the material's rheological behaviour. In general, this rheological behaviour can be classified by five different material types as shown in Fig. 1. For Newtonian fluids, the shear stress $\tau$ has a linear correlation with the shear rate $\gamma$ in which the slope of the line is the constant Newtonian viscosity $\mu$. The second type is represented by pseudoplastic fluids that are often represented by the Ostwald-de Waele power law equation. The third one is named dilatant fluids, and they are characterised by an increasing slope of the shear stress-shear rate behaviour. The fourth is the Bingham plastic material which has a yield point $\tau_{\mathrm{y}}$ below which no flow takes place, whereas above it, the behaviour is linear and characterised by the plastic viscosity. Finally, for viscoplastic materials, there is also a yield point like for the Bingham material, but above that, a polynomial behaviour can be seen.

The first model for predicting the tape thickness in tape casting was developed by Chou et $a l^{4}$ In their work, the Newtonian behaviour was used and they assumed that the flow in the parallel doctor blade region is a linear combination of pressure and drag flow. However, in the tape casting process of ceramics, the slurry seldom behaves like a Newtonian fluid. Pitchumdni and Karbhari ${ }^{9}$ evaluated the effects of an imposed pressure gradient due to the height of the slurry in the casting head, as well as those of the drag due to the moving substrate on the slurry flow by modelling the slurry discharge as a generalised power law flow, i.e. 


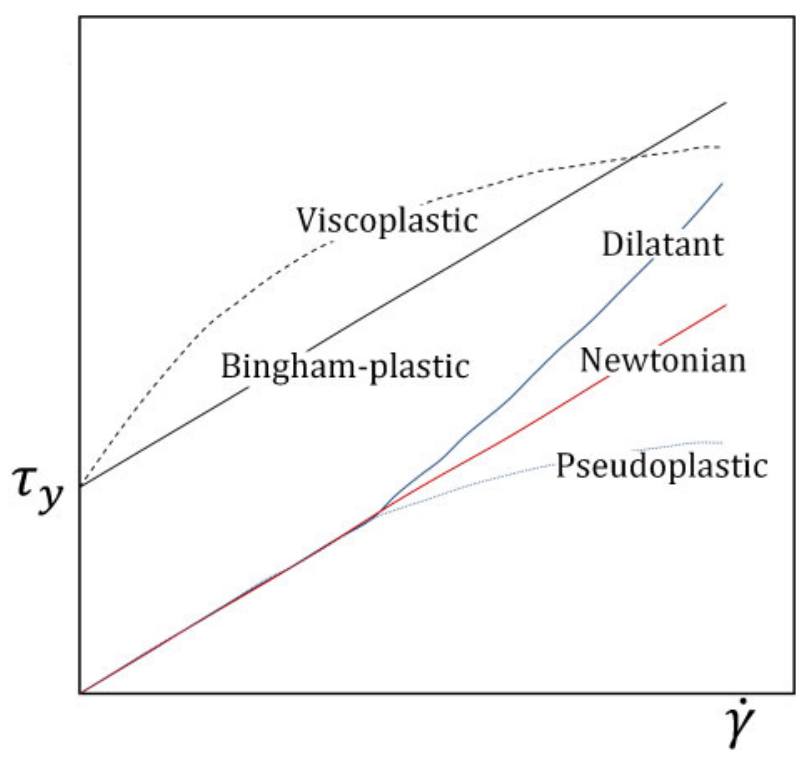

1 Rheological classification of flow

$$
\tau=k \dot{\gamma}^{\mathrm{n}}
$$

where $k$ and $n$ are the consistency of the fluid and deviation from a Newtonian fluid respectively, and both are constants for a specific slurry. However, in their work, the height of the ceramic slurry is assumed to be constant as opposed to the present work where it is allowed to vary.

Ring ${ }^{10}$ modelled the tape casting slurry by applying the Bingham plastic constitutive law

$$
\tau=\tau_{0}+\mu \gamma
$$

where $\tau_{0}$ is the Bingham yield stress, which is the finite stress required for flow initiation, and $\mu$ is the plastic viscosity. In this model, the material acts as a rigid body below the yield point, and above it, the ceramic slurry flows with the constant plastic viscosity. However, the $\left(\mathrm{La}_{0.85} \mathrm{Sr}_{0 \cdot 15}\right)_{0.9} \mathrm{MnO}_{3}$ (LSM) ceramic used in the present work did not show any yield point, which means that the slurry flows all the time. Recently, the Herschel-Bulkley model was used in the tape casting process for modelling the flow field by Huang et al. ${ }^{11}$ This model is a combination of the Bingham plastic and power law models; however, again, they did not consider the transient behaviour of the ceramic slurry height.

Ceramics might be attractive materials for high temperature applications provided that both their reliability and toughness could be improved. The key factor improving the toughness of these materials is the presence of weak interfaces between fibres and the composite matrix or between the ceramic layers in multilayered structures. These interfaces allow for energy dissipation before fracture through mechanisms of crack deflection, crack bridging, fibre pullout and interface delamination. Multilayered ceramics are generally processed by tape casting and firing or hot pressing. ${ }^{12}$ The presence of the different layers in multilayer materials controls the crack path, frequently avoiding completely brittle behaviour. When the layers are strongly bonded together, a crack in one layer can propagate readily into the adjacent layer, and then the material behaves as a conventional ceramic. ${ }^{13}$ Most often, the graded structures are produced from laminating two or more single layers. In this respect, controlling the tape thickness and its uniformity along the casting direction becomes more important.

Moreover, the shape changes that happen during the sintering process due to the shrinkages in different directions will result in shape instability. ${ }^{14}$ This shape instability is more important in the multilayer materials, since during the sintering process every layer has different thermal behaviour. However, even in the case of monolayer tape casting, it is of great importance to control the aforementioned shape instabilities. Raj and Cannon ${ }^{14}$ proposed a formula to measure the percentage of anisotropic shrinkage in the tape casting process. They showed that the tape thickness and its variation have a great impact on the anisotropic shrinkage and the resultant mechanical properties, where an increase in the tape thickness causes a decrease in the anisotropic shrinkage. This emphasises the importance of the thickness control in the tape casting process.

Hence, in the present paper, an analytical model capable of predicting the final tape thickness is presented. It is based on a quasi-steady state description of the velocity and the pressure field in the doctor blade region, allowing the slurry height to drop during casting, and this is combined with a power law model for the fluid flow. Many of the affecting parameters in the process are embedded and they can easily be varied to evaluate their influence. The proposed models describe the flow characteristics of tape casting well. Results of the model are compared with experiments, and good agreement is obtained.

In the present paper, a quasi-steady state description of the velocity and the pressure field in the doctor blade region, allowing the slurry height to drop during casting, is combined with a power law model for the fluid flow. Many of the affecting parameters in the process are embedded, and they can easily be varied to evaluate their influence. The proposed models describe the flow characteristics of tape casting well. Results of the model are compared with experiments, and good agreement is obtained.

\section{Analysis}

In order to express the volume flow and thus the tape thickness, the velocity field equation in the doctor blade region must be developed. Based on the number of doctor blades, there are two kinds of tape casters: a simple tape caster that has only one doctor blade (illustrated schematically in Fig. 2), and a double blade tape caster in which a front doctor blade is used to ensure a hydrostatic pressure in front of the rear one. ${ }^{15}$

As shown in Fig. 2, $\delta$ is the green tape thickness, $h$ is the doctor blade height, $W$ is the width of the doctor blade, $H_{0}$ is the height of the slurry in front of the doctor blade, $d$ is the depth of the reservoir, $l$ is the inclined length of the reservoir and $\theta$ is the angle of the reservoir.

The pressure gradient inside the channel below the doctor blade is constant, since there is a hydrostatic pressure in front of the doctor blade and it can be determined by the height of the slurry as shown below ${ }^{15}$

$$
\frac{\mathrm{d} p}{\mathrm{~d} x}=-A_{0}=-\frac{\rho g H_{0}}{W}
$$

where $\rho$ is the density of the slurry, and $g$ is the acceleration due to gravity. 


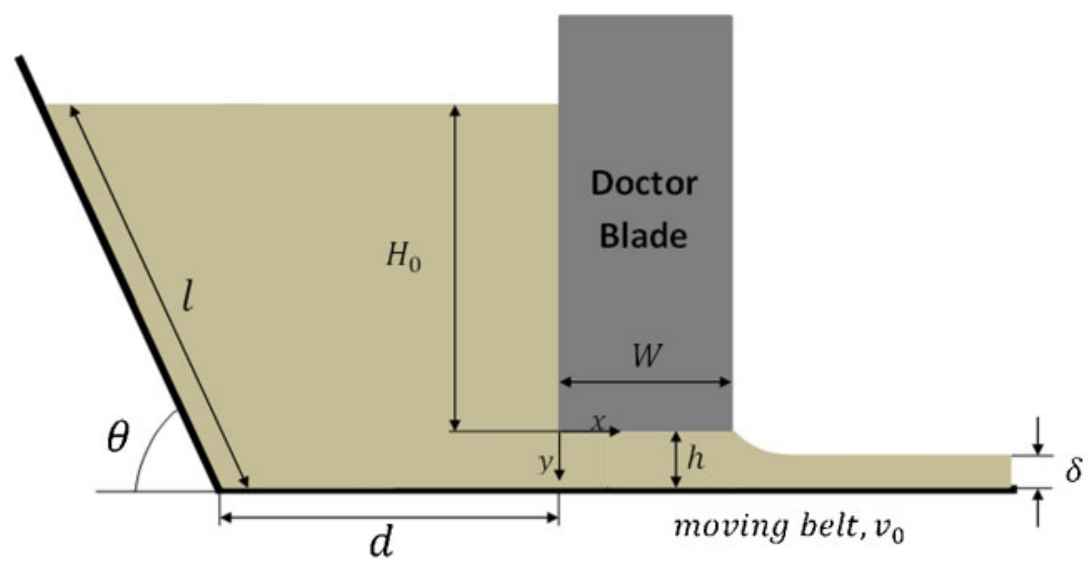

2 Schematic geometry of tape casting machine

By assuming an infinitely long and wide plate as compared to the thickness and combining with momentum conservation in the $x$ direction under steady state conditions, we obtain the following ${ }^{15,16}$

$$
\frac{\mathrm{d} \tau}{\mathrm{d} y}=\frac{\mathrm{d} p}{\mathrm{~d} x}
$$

where $\tau$ is the shear stress. From equations (3) and (4), $\tau$ is found to be

$$
\tau=-A_{0} y+A_{1}
$$

where $A_{1}$ is an integration constant.

For power law fluids, the shear stress $\tau$ is given by the constitutive equation $^{16}$

$$
\tau=k\left(\frac{\partial u}{\partial y}\right)^{\mathrm{n}}
$$

where it has been assumed that the only velocity component contributing to the shear rate is the velocity in the $x$ direction $u$.

Rewriting equations (5) and (6) and integrating along the channel height $h$

$$
\int_{0}^{\mathrm{h}}\left(-A_{0} y+A_{1}\right) \mathrm{d} y=\int_{0}^{\mathrm{h}} k\left(\frac{\partial u}{\partial y}\right)^{\mathrm{n}} \mathrm{d} y
$$

and assuming that $k$ and $n$ are constants, we obtain

$u=\left(-\frac{1}{A_{0} k \frac{1}{n}}\right)\left(\frac{1}{\frac{1}{n}+1}\right)\left(-A_{0} y+A_{1}\right)^{\frac{1}{n}+1}+A_{2}(0<y<h)$

where $A_{2}$ is another integration constant.

The boundary conditions for equation (8) in the doctor blade region of tape casting are

$$
\left\{\begin{array}{l}
u(0)=0 \\
u(h)=v_{0}
\end{array}\right.
$$

where $v_{0}$ is the velocity of the moving belt.

Applying these boundary conditions in equation (8), and introducing the expressions that $(1 / n)+1=\chi$ and $-\left[1 / A_{0} k(1 / n)\right]=\psi$, we get

$$
\left\{\begin{array}{l}
0=\frac{\psi}{\chi} A_{1}^{\chi}+A_{2} \\
v_{0}=\frac{\psi}{\chi}\left(-A_{0} h+A_{1}\right)^{\chi}+A_{2}
\end{array}\right.
$$

which is rewriting into

$$
\left\{\begin{array}{l}
v_{0}=\frac{\psi}{\chi}\left[\left(-A_{0} h+A_{1}\right)^{\chi}+A_{1}^{\chi}\right] \\
A_{2}=-\frac{\psi}{\chi} A_{1}^{\chi}
\end{array}\right.
$$

In order to find the integration constant $A_{1}$, the Newton-Raphson method is used. To do so, the following equation originating from the upper expression in equation (11) is solved numerically to find the parameter $A_{1}$

$$
F\left(A_{1}\right)=\frac{\psi}{\chi}\left[\left(-A_{0} h+A_{1}\right)^{\chi}+A_{1}^{\chi}\right]-v_{0}=0
$$

and consequently after finding $A_{1}$, the parameter $A_{2}$ is calculated from the lower expression in equation (11).

As a consequence of mass conservation, the thickness of the green tape $\delta$, can then be determined by integrating $u$ over the channel height and dividing by the tape velocity, i.e.

$$
\delta=\frac{1}{v_{0}} \int_{0}^{\mathrm{h}} u \mathrm{~d} y=\frac{-\left[\left(-A_{0} h+A_{1}\right)^{\chi+1}-A_{1}^{\chi+1}\right]}{v_{0} A_{0} \psi \chi(\chi+1)}+A_{2} h
$$

Reaching to a constant tape thickness is not an impossible goal, since most of the manufacturing processes for tape casting are at the continuous form in which the reservoir at all times is fed by slurry. Moreover, using two doctor blades in the design of the machine will result in having almost constant hydrostatic pressure during the casting process. However, it is of great importance to control the tape thickness in the small tape casters especially in small scale production and laboratories, in which the slurry height is not constant in the reservoir but gradually decreasing with time. This phenomenon leads to the present modification of the standard steady state model, ${ }^{2,4,9,15,16}$ making it dependent on the height variation.

Now, the proposed quasi-steady state formulation is developed based on the continuity equation. Assuming that the slurry is incompressible, the volume of the slurry which drops down in the reservoir is equal to the volume of the slurry which is conveyed out of the doctor blade region by the peeling belt. Assuming that during the time period equal to $\Delta t$, the height of the slurry will decrease from the initial value of $H_{0}$ to $H_{1}$, the aforementioned volume (equals area in the present 
two-dimensional model) in the reservoir which is decreased will be given as

$$
\Delta S_{1}=\left[d+\frac{1}{2} l \cos \theta\left(1+\frac{H_{1}+h}{H_{0}+h}\right)\right]\left(H_{0}-H_{1}\right)
$$

This area is moved out of the doctor blade region with the constant velocity of $v_{0}$ and the distance of $v_{0} \times \Delta t$; hence, it is equal to $v_{0} \times \Delta t \times \delta_{1}$. The new initial height in the next time step is now $H_{1}$ and the new $\Delta S_{2}$ is found from using $H_{1}$ as initial height in equation (14) and so forth.

In the tape casting process, when the flow exits the doctor blade region, the fluid starts to flow in a transverse direction also (here in the $z$ direction) and this is mostly named side flow. As the final tape is in general very wide in the $z$ direction compared to its thickness, this side flow will be relatively small. Moreover, after the drying stage, the tape thickness is decreased due to weight loss. Considering both these effects, the final thickness of the dried tape is

$$
\delta_{\mathrm{tp}}=\frac{\alpha \beta \rho}{\rho_{\mathrm{tp}}} \delta
$$

where $\delta_{\mathrm{tp}}$ is the thickness of the dried tape, $\alpha$ is the fraction loss for side flow, $\beta$ is the fraction loss for weight reduction due to drying, $\rho$ is the density of green tape and $\rho_{\text {tp }}$ is the density of dried tape, and $\delta$ is the thickness of the green tape.

\section{Experimental}

The different constituents and their function for the LSM slurry used in the experiments are given in Table 1. A commercial LSM was calcined at $1000^{\circ} \mathrm{C}$ and used in the amount of $62.05 \mathrm{wt}-\%$ in the final slurry. The average particle size and specific surface area were $12 \cdot 42 \mu \mathrm{m}$ and $23 \cdot 24 \mathrm{~m}^{2} \mathrm{~g}^{-1}$ respectively. Moreover, the weight ratios between LSM and MEKET (solution of methylethylketone and ethanol with the weight ratios of $1: 3$ ) and PVB/LSM were $1: 2.44$ and $1: 11.53$ respectively. To remove air bubbles from the slurries, mechanical vacuum was enforced for $15 \mathrm{~min}$. The mean particle size and density of the final slurries were accordingly equal to $2 \cdot 20-2 \cdot 73 \mu \mathrm{m}$ and $1.91 \mathrm{~g} \mathrm{~mL}^{-1}$. The particle size distributions were measured with a laser diffraction particle size analyser LS 13320 from Beckman Coulter (USA). The rheological profiles were measured using a narrow gap parallel plate sensor system in Rheometer HAAKE Rheo Stress 600 (Haake, Germany).

Experiments were carried out using a one-doctor blade continuous type of tape casting bench, and the

Table 1 Material content and their function for LSM slurry used in experiments

\begin{tabular}{ll}
\hline Material & Function \\
\hline$\left(\mathrm{La}_{0.85} \mathrm{Sr}_{0 \cdot 15}\right)_{0.9} \mathrm{MnO}_{3}(\mathrm{LSM})$ & Ceramic substrate \\
Methylethylketone & Solvent \\
Ethanol & Solvent \\
Polyvinyl pyrrolidone (PVP) & Dispersant \\
Polyvinyl butyral (PVB) & Binder \\
Polyethylene glycol (PEG) & Plasticiser \\
Dibutyl phthalate (DBP) & Plasticiser \\
Additol & Deflocculant \\
\hline
\end{tabular}

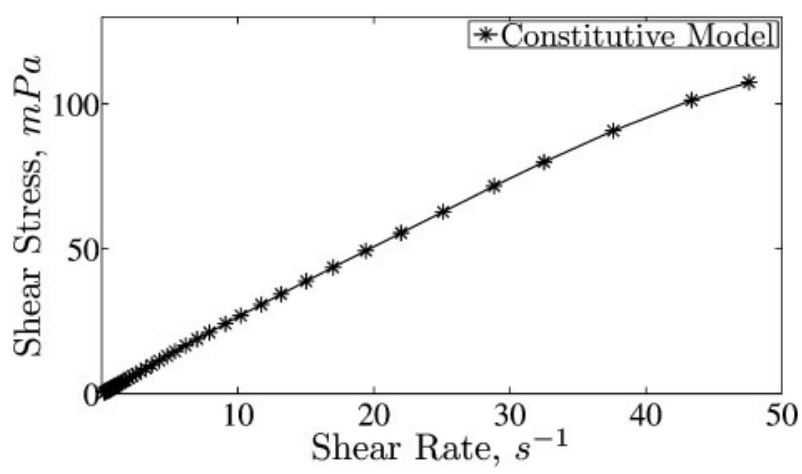

3 Rheology behaviour of LSM slurry

plastic carrier tape (Mylar in the present case) was driven by stainless steel rollers. The doctor blade gap distance was set by using etalon sticks and a micrometre screw with an accuracy of $0.01 \mathrm{~mm}$. After the propulsion of the torque drum, whose speed was programmed in advance, the slurry was peeled out according to the carrier movement, forming a thin layer of tape. The cast tapes were dried in air for 5 days, and their thicknesses were measured using the micrometer screw. The thicknesses of the cast tapes were measured in every $10 \mathrm{~cm}$ from doctor blade exit.

Experiments were conducted in three different cases, which are summarised in Table 2. For the first set of experiments, the same amount of ceramic slurry (constant $H_{0}$ ) was cast with three different substrate velocities. Then, in case 2 , with constant velocity and slurry height, the amount of the doctor blade height was varied. In the last set of experiments during constant substrate velocity, the material load in the reservoir $H_{0}$ was varied. These experiments were carried out to investigate the effect of the three important parameters, i.e. drag forces related to substrate velocity, hydrostatic pressure and doctor blade height on the side flow amount.

\section{Results and discussion}

Results from the rheology experiment showed that the LSM slurry follows the Ostwald-de Waele power law fluid behaviour. This is illustrated in Fig. 3, and the relationship for the shear rate and shear stress was found to be

$$
\tau=3 \cdot 31(\partial u / \partial y)^{0 \cdot 90}
$$

where the constant $k$, which is the consistency of fluid, is equal to $3.31 \mathrm{mPa} \mathrm{s}$ (where $n=0 \cdot 90$ ), and the constant $n$, which is the proximity to a Newtonian fluid, is equal to $0 \cdot 90$, quite close to the Newtonian fluid value of 1 . The constant for the side flow $\alpha$ was obtained by a volumetric comparison of the tape which flowed outside

\section{Table 2 Summary of experimental studies}

\begin{tabular}{llll}
\hline Parameter & Case 1 & Case 2 & Case 3 \\
\hline$W / \mathrm{mm}$ & $6 \cdot 4$ & $6 \cdot 4$ & $6 \cdot 4$ \\
$h / \mathrm{mm}$ & 1 & $0 \cdot 25,0 \cdot 4,1$ & $0 \cdot 4$ \\
$v_{0} / \mathrm{mm} \mathrm{s}^{-1}$ & $3 \cdot 67,10,14 \cdot 67$ & $3 \cdot 67$ & $3 \cdot 67$ \\
$H_{0} / \mathrm{mm}^{\prime}$ & $1 \cdot 2$ & $10 \cdot 6$ & $3 \cdot 1,6 \cdot 6,20 \cdot 8$ \\
$d / \mathrm{cm}$ & $8 \cdot 8$ & $8 \cdot 8$ & $8 \cdot 8$ \\
$\alpha{ }^{\circ}$ & 45 & 45 & 45 \\
\hline
\end{tabular}




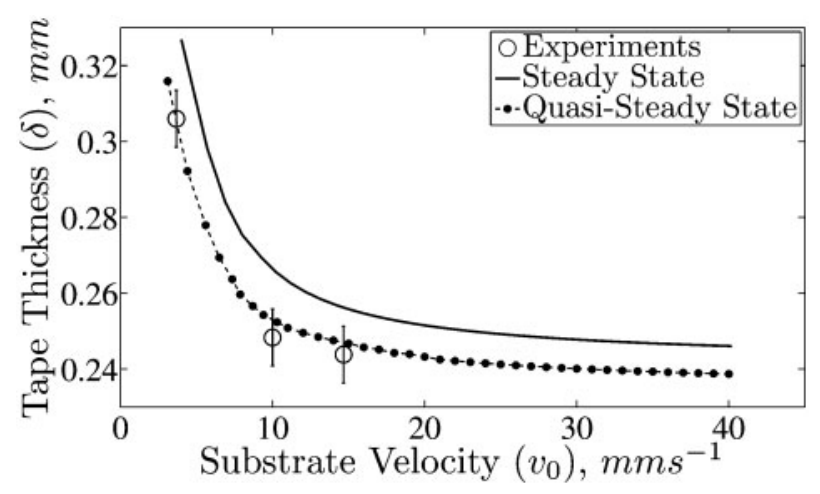

4 Effect of substrate velocity on tape thickness with doctor blade height of $1 \mathrm{~mm}$

the casting width to the tape within the casting width $(0 \cdot 8<\alpha<0 \cdot 9)$. The drying weight loss factor $\beta$ was obtained from drying experiments, which measured the dried tape mass and compared it to the wet slurry mass $(0 \cdot 58<\beta<0 \cdot 64)$.

\section{Effect of substrate velocity}

Figure 4 shows the effect of the substrate velocity on the dried tape thickness for the experimental data, steady state and quasi-steady state model. As seen from the figure for all types of data, an increased substrate velocity results in decreasing of the tape thickness. By further increase in the substrate velocity, it is found that the tape thickness decreases hyperbolically, ${ }^{4,9}$ which is also seen from equation (13). From previous works, ${ }^{4,8,9}$ it was found that when the drag force is increased by increasing the substrate velocity, it becomes more dominant compared to the pressure force that results in more stretching of the slurry over the peeling belt. Figure 4 shows that the proposed quasi-steady state model is in better agreement with corresponding experiments as compared to the steady state model, since in the quasi-steady state model the effect of decreasing level of the slurry height was taken into account. It should be noted that the variation of the height in the slurry will change the parameter $A_{0}$ and the resultant values of $A_{1}$ and $A_{2}$ (which are calculated numerically); this of course is neglected in the steady state model.

Figure 5 depicts the velocity profiles below the doctor blade for three different tape velocities. It is seen that for the higher substrate velocity (of $14.67 \mathrm{~mm} \mathrm{~s}^{-1}$ ), the distribution approaches a linear correlation, which

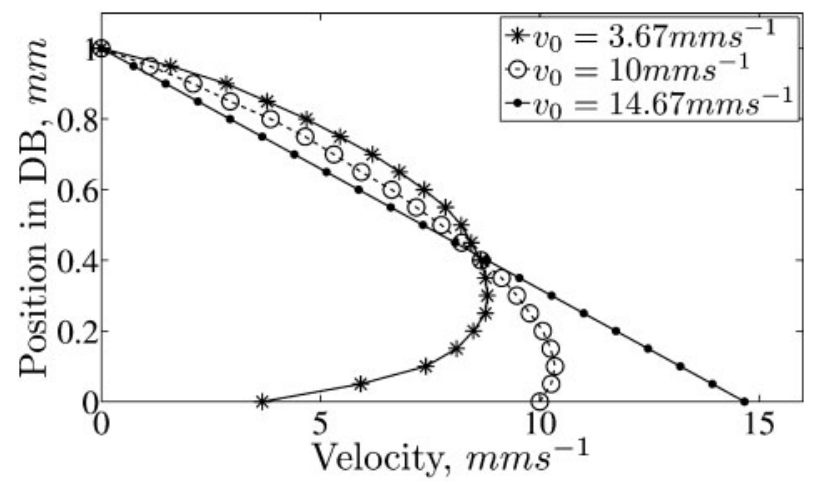

5 Average velocity profile below doctor blade region with different substrate velocities

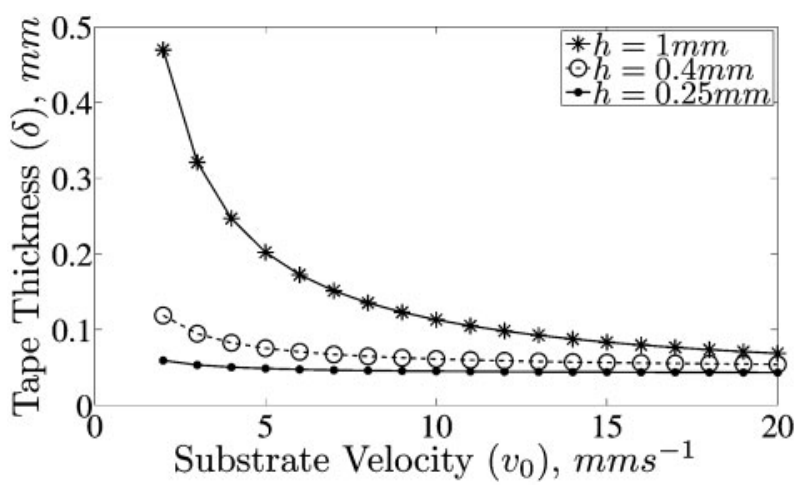

6 Results of modelling for effect of doctor blade height on tape thickness

corresponds to Couette flow conditions. For lower velocities, the hydrostatic pressure plays a more dominant role, resulting in a velocity peak $\sim 0.25 \mathrm{~mm}$ above the peeling belt, which originates from the combination of Couette and Poiseuille flow conditions. These tendencies are also found in the analytical model for flow in tape casting originally proposed by Kim et $a .^{2}$ and further developed on dimensionless form by Jabbari and Hattel. ${ }^{8}$

\section{Effect of doctor blade height}

The effect of the doctor blade height on the tape thickness is illustrated in Fig. 6. It is seen that increasing the doctor blade height leads to a higher tape thickness, which is expected. However, the difference between the curves in Fig. 6 is decreased by increasing the velocity. For a constant pressure height, increasing the doctor blade height results in more material being carried out of the doctor blade region. This means that the thickness of the tape will be increased. As mentioned before, for high casting velocities (substrate velocity), the drag force becomes more dominant. The increase in the tape thickness coming from a higher doctor blade gap is reduced as the velocity is increased.

The comparison between the proposed model, steady state model and experimental results is shown in Fig. 7. It can be seen that the new proposed model is in better agreement with experiments in comparison to the steady state model. The highest deviation between data was observed for the higher values of the doctor blade height. This could arise from some of the assumptions used in the equations for the proposed model. The main assumption in this regards is the use of constant side

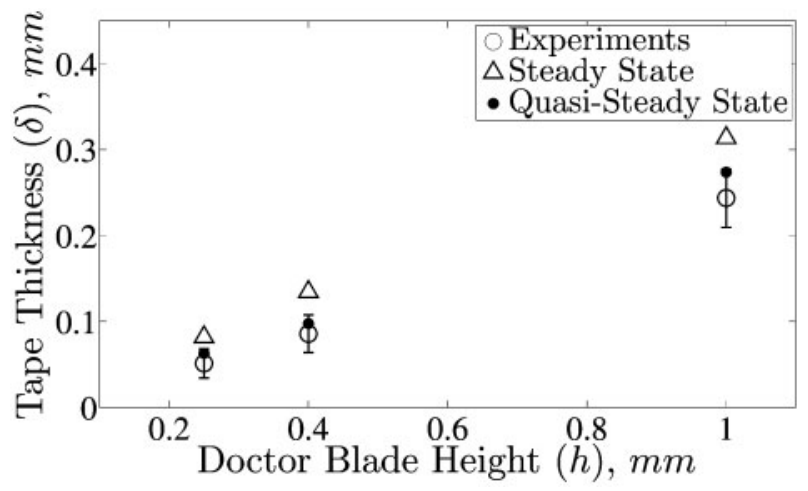

7 Comparison of proposed quasi-steady state model with steady state model and experiments for $v_{0}=3.67 \mathrm{~mm} \mathrm{~s}^{-1}$ 


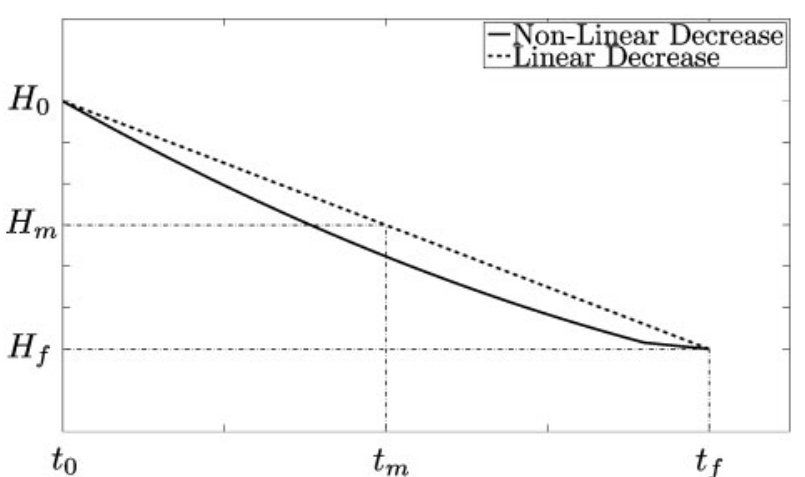

8 Schematic illustration of slurry height change by time

flow factor $\alpha$ for calculating the tape thickness based on equation (15). However, this factor is not constant based on the numerical investigation done by the authors, ${ }^{17}$ and highly dependent on the geometry of the tape caster and the process parameter. On the other hand, in most cases, the desired thickness for the final tapes is in the range of $\leq 100 \mu \mathrm{m}$, in which the proposed model agrees well with the experimental data. The smaller deviation found in the experiment might be due to the effect of side flow, which obviously reduces the tape thickness as compared to the analytical solution.

\section{Effect of slurry load}

It should be emphasised that for the comparisons shown in Figs. 4 and 7, the height of the slurry that was inserted in the steady state solution ${ }^{15,16}$ was the initial height $H_{0}$. This of course overestimates the hydrostatic pressure and hence the tape thickness in cases where the slurry height is actually decreasing over time.

Thus, in order to make a more fair comparison between the analytical steady state model and the proposed quasi-steady state model, it was chosen to represent the slurry height (which is constant) in the steady state model by some reasonable average.

Now, consider Fig. 8 in which the decrease in the height of the slurry in the reservoir over time is represented schematically. Here, $H_{0}$ is the initial ceramic height and $H_{\mathrm{f}}$ is the last point where the fluid experiences the hydrostatic pressure (equal to the doctor blade height $h$ ). Of course, the real trend for the decrease of the height is a non-linear behaviour, starting with high hydrostatic pressure and decreasing by time, but as a reasonable average, one could assume that there is a linear behaviour in the reduction of the slurry height by time, i.e.

$$
H_{\mathrm{m}}=\frac{H_{0}+H_{\mathrm{f}}}{2}
$$

This mean value is used in the steady state model when comparing with the quasi-steady state model in Figs. 9 and 10 .

By choosing fairly small time increments for the modelling, the results for different values of heights in the reservoir are shown in Fig. 9. As it seen from Fig. 9, the thickness of the tape in the end of a strip is higher than that in the beginning of a strip. This is due to the higher level of material in the reservoir in the beginning of the process. As time passes, the height of the slurry in the reservoir decreases, and consequently, the resultant height of the tape will decrease. This means that the hydrostatic pressure decreases by time and the drag forces start to show their dominance by making the tape thinner. However, this phenomenon cannot be detected by the steady state model, no matter which value of the slurry height (initial or average) is applied. Moreover, it can be seen that the higher level of slurry in the reservoir leads to a higher tape thickness in the strip. On the other
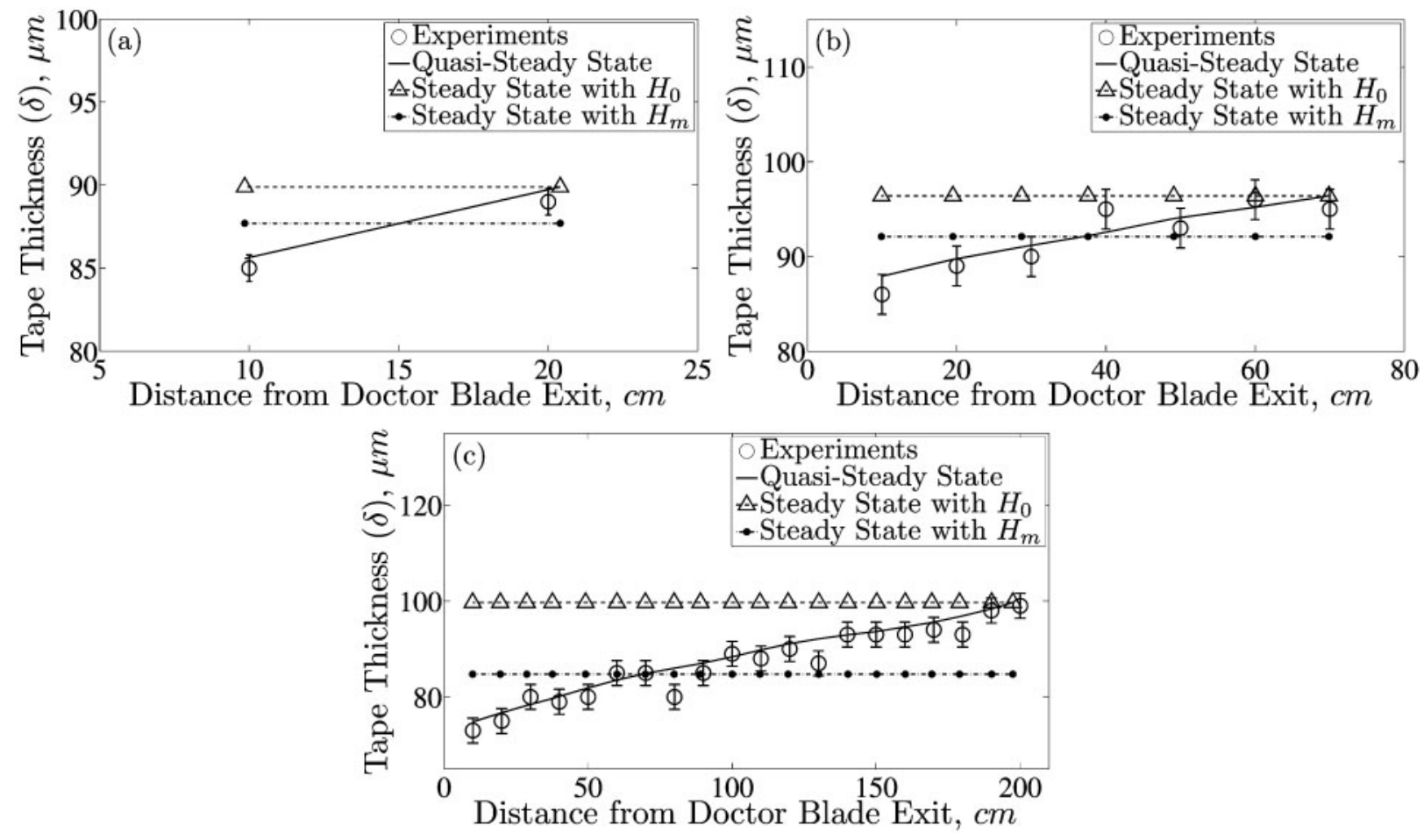

a $H_{0}=3.1 \mathrm{~mm} ; b H_{0}=6.6 \mathrm{~mm} ; c H_{0}=20.8 \mathrm{~mm}$

9 Results of modelling and their comparison with experimental data for case 3 


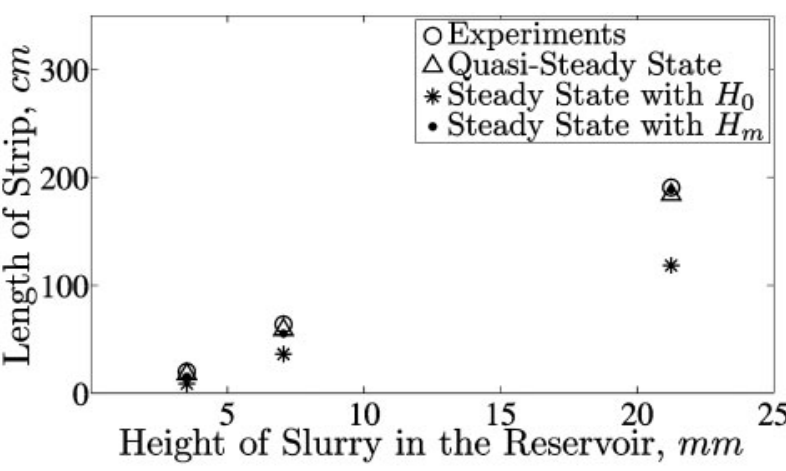

10 Correlation between height of slurry and length of strip from model and experiments with substrate velocity of $3.67 \mathrm{~mm} \mathrm{~s}^{-1}$

hand, it is also seen that the mean assumption for the initial slurry height (steady state with $H_{\mathrm{m}}$ ) in some points catches the experimental data better than the one with the initial height (steady state with $H_{0}$ ). However, it does not predict the transient behaviour of the slurry height, which is embedded in the quasi-steady state model.

Furthermore, the higher level of slurry will result in a longer final strip because of more material content in the process. The predicted values from the quasi-steady state, steady state model with initial height and steady state with the mean height assumption and the estimated data from experiments for the length of strip with the different heights of the slurry are shown in Fig. 10. The results of the quasi-steady state model are in very good agreement with the experimental data. The small differences between the experiments and the proposed model might be due to the influence of the side flow. However, it can also be seen that there is much more difference between the steady state model and the experimental data, which was expected. It is obvious that the steady state model with the initial height gives a constant value of the tape thickness, which only fits to the experimental and the quasi-steady state model in the beginning where the slurry height is actually $H_{0}$. Moreover, the results of the steady state model with the mean assumption for the initial slurry height are in good agreement with both the quasi-steady state and the experimental data; however, they are still constant and a more representative level is expected.

To get a better understanding of the effect of the variable slurry height on the tape thickness, the velocity profile was analysed below the doctor blade region for one of the tests in case $3\left(H_{0}=20.8 \mathrm{~mm}, h=0.4 \mathrm{~mm}\right.$ and $v_{0}=3.67 \mathrm{~mm}$ ) which is shown in Fig. 11. Here, the line with the asterisk marker represents the velocity profile for the initial slurry height $\left(H_{0}=20.8 \mathrm{~mm}\right)$, the line with the 'o' marker is the representative of the velocity profile after some period of time and the line with the dot marker represents results even later in time. As seen, since the hydrostatic pressure decreases by time due to

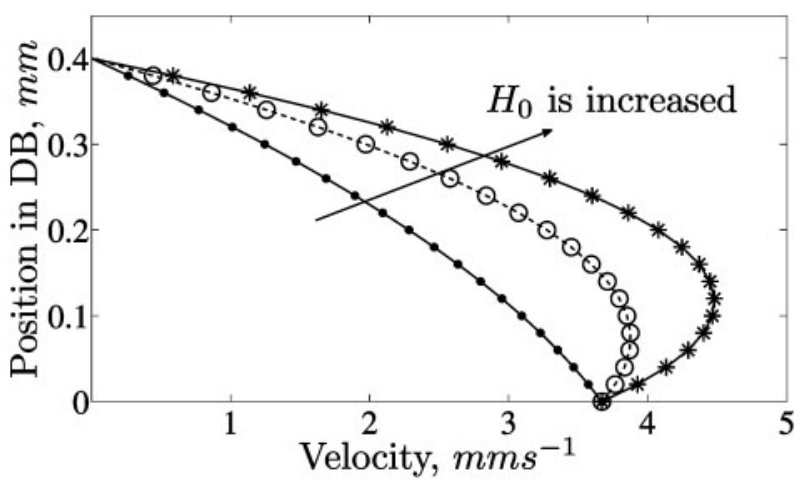

11 Effect of variation in slurry height on velocity profile below doctor blade region with doctor blade size of $h=0.4 \mathrm{~mm}$

the reduction in level in the slurry height, the velocity profile is changed and the area under the velocity profile is decreased, which can be seen in Fig. 12, and consequently, the 'area' out of the blade is decreased. On the contrary, decreasing the pressure head for a constant velocity, the thickness of the tape is decreased in the exit and vice versa. This behaviour very much emphasises the importance of the proposed quasi-steady state model, in which the transient effect of the slurry height in the reservoir (which resembles the pressure head) is implemented. As already discussed, the variation in the velocity profile will cause a change in the tape thickness (Fig. 12). This phenomenon can easily be seen in Fig. 9, where the tape thickness decreases in the casting direction due to the transient decrease of the slurry height in the reservoir.

\section{Conclusions}

A quasi-steady state power law model for the constitutive behaviour of the non-Newtonian slurry of LSM was proposed and used to analyse the effect of substrate velocity, doctor blade height and slurry height in the reservoir on the final tape thickness in tape casting. This proposed model was based on the continuity equation assuming incompressibility such that the decrease of the volume of the slurry in the reservoir is equal to the one that leaves the doctor blade region. The results show that increasing the substrate velocity (casting speed) causes a decrease in the tape thickness due to the dominance of drag force over hydrostatic pressure. On the other hand, increasing the doctor blade height with constant velocity, the thickness of the final tape will be increased, since the gap size for the slurry is high enough for it to be conveyed more out of the doctor blade region. In both cases, the developed quasi-steady state model has better agreement with the experiments compared to the well known steady state model. For both quasi-steady state model and experiments, it is observed that the height of tape at the end point of the strip is higher in comparison to the beginning of the
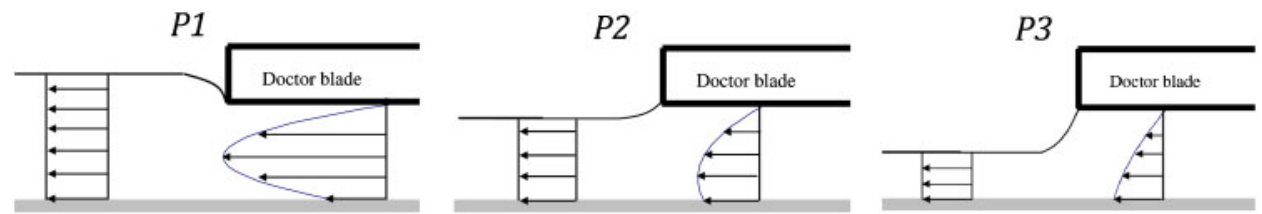

12 Schematic illustration of effect of increased pressure head on tape thickness $\left(P_{1}>P_{2}>P_{3}\right)^{2}$ 
strip, which is not predictable by steady state solution. Moreover, by increasing the material load (the height of slurry in the reservoir), the aforementioned differences between the beginning and the end of strip and the length of strip will be increased. A new modified steady state model is also presented based on a linear correlation between the level in the slurry height and the time. Although the presented model does not have the full accuracy of the quasi-steady state model, it showed some good results compared to the conventional steady state calculations from the literature. Although the LSM ceramic used in the present study has a slightly nonNewtonian behaviour $(n=0.90)$, the model contains all main parameters that influence the process, and it has the flexibility to be used for different slurries, which are more non-Newtonian, as well as different machine designs. Moreover, the results show that the classical steady state is not so well describing for the real process in which the ceramic height in the reservoir is not constant. However, the results of the proposed quasi-steady state model show that it has good agreement with the experimental data.

\section{Acknowledgements}

The authors would like to acknowledge the support of the Scientific Research Councils on Technology and Production Sciences (FTP) (contract no. 09-072888, OPTIMAC), which is part of the Danish Council for Independent Research (DFF).

\section{References}

1. G. S. Grader and L. Zuri: J. Am. Ceram. Soc., 1993, 76, 1809-1814.

2. H. J. Kim, M. J. M. Krane, K. P. Trumble and K. J. Bowman: J. Am. Ceram. Soc., 2006, 89, 2769-2775.

3. C. Pagnoux, T. Chartier, M. Granja, F. Doreau, J. M. Ferreira and J. F. Baumard: J. Eur. Ceram. Soc., 1998, 18, 241-247.

4. Y. T. Chou, Y. T. Ko and M. F. Yan: J. Am. Ceram. Soc., 1987, 70, 280-282.

5. Y. Tanimoto, T. Hayakawa and K. Nemoto: Dent. Mater., 2007, 23, 549-555.

6. M. P. Albano and L. B. Garrido: Ceram. Int., 2005, 31, 57-66.

7. P. H. Gaskell, B. Rand, J. L. Summers and H. M. Thompson: J. Eur. Ceram. Soc., 1997, 17, 1185-1192.

8. M. Jabbari and J. Hattel: AIP Proc. Int. Conf. on 'Numerical analysis and applied mathematics', Halkidiki, Greece, American Institute of Physics (AIP), September 2011, Vol. 1389, 143-146.

9. R. Pitchumdni and V. M. Karbhari: J. Am. Ceram. Soc., 1995, 78, 2497-2503.

10. T. A. Ring: Adv. Ceram., 1989, 26, 569-576.

11. X. Y. Huang, C. Y. Liu and H. Q. Gong: Mater. Manuf. Processes, 1997, 12, 935-943.

12. C. Badinia, P. Fino, A. Ortona and C. Amelio: J. Eur. Ceram. Soc., 2002, 22, 2071-2079.

13. C. A. Folsom, F. W. Zok and F. F. Lang: J. Am. Ceram. Soc., 1994, 77, 689-696.

14. P. M. Raj and W. R. Cannon: J. Am. Ceram. Soc., 1999, 82, 26192625.

15. G. Zhang, Y. Wang and J. Ma: Mater. Sci. Eng. A, 2002, A337, 274-280.

16. A. I. Y. Tok, F. Y. C. Boey and Y. C. Lam: Mater. Sci. Eng. A, 2000, A280, 282-288.

17. M. Jabbari and J. H. Hattel: J. Am. Ceram. Soc., 2013, 96, 1414 1420 . 